\title{
Postnatal Strategies to Prevent Bronchopulmonary Dysplasia: An Update
}

\author{
Roosy Aulakh* \\ Associate Professor, Department of Pediatrics, Government Medical College and Hospital, Chandigarh, India \\ *Corresponding Author: Roosy Aulakh, Associate Professor, Department of Pediatrics, Government Medical College and Hospital, \\ Chandigarh, India.
}

Received: October 30, 2019; Published: November 19, 2019

DOI: $10.31080 /$ ASPE.2019.02.0185

\begin{abstract}
Bronchopulmonary Dysplasia (BPD) is a leading cause of morbidity and mortality in neonates especially with rising preterm deliveries across the world due to advancements in obstetric medicine and neonatal care. Multiple risk factors are known to contribute to development of BPD in premature neonates which have opened multiple potential targets to develop innovative preventative and treatment strategies for BPD in the recent decades.

Keywords: Bronchopulmonary Dysplasia; Oxygen; Lung Disease
\end{abstract}

\section{Definition}

BPD was first described in 1967 by Northway., et al. in an era when the mortality from respiratory distress syndrome (RDS) was $>50 \%$ [1]. There has been an ongoing evolution in the understanding of BPD and also the profile of BPD is changing due to changes in neonatal management strategies like use of surfactant, non invasive mechanical ventilation and corticosteroids. This has led to development of numerous definitions for BPD. The most commonly used definition for BPD has been the one proposed in 2001 by Jobe AH., et al. who proposed severity-based diagnostic criteria for BPD that included oxygen need for 28 days and an assessment of respiratory support at 36 weeks' postmenstrual age [2]. However, numerous pitfalls have been reported with this definition of BPD and modifications proposed over the years. New criteria have been proposed to include infants dying of severe respiratory failure prior to 36 weeks' PMA (severe BPD), to categorize infants on different flows of nasal cannula oxygen, and provide specific guidance for including only infants with significant parenchymal lung disease $[3,4]$.

\section{Strategies for prevention of BPD}

The current review will be restricted to the postnatal strategies in prevention of BPD, detailed as under;

Use of corticosteroids

- Mechanism of action: The anti-inflammatory action of corticosteroids is complex. At a cellular level, they cause redistribution of granulocytes, resulting in increased circulating granulocytes and reduced tissue pools. They also cause lymphopenia. They cause adrenergically mediated vasoconstriction and non-competitive antagonism of vasodilation due to prostaglanin $\mathrm{E}$ and bradykinin. Prostaglandin formation is inhibited by corticosteroids.
Corticosteroids stabilize the lysosomal membrane preventing release of lysosomal enzymes in vitro but the significance of this in vivo is debatable [5].

- Basis of use in BPD: As BPD is considered to be a result of inflammation, the use of corticosteroids to suppress inflammation has been proposed to prevent BPD.

- Route of administration: Intravenous.

- Preparations: Dexamethasone/hydrocort.

- Adverse Effects: Short term (hyperglycemia, hypertension, gastrointestinal bleeding, gastrointestinal perforation, hypertrophic cardiomyopathy, increased risk of infection) and long term (neuro-developmental especially cerebral palsy) [6].

- Current Status: American Academy of Pediatrics (AAP) Committee on Fetus and Newborn and the Canadian Paediatric Society (CPS) recommend that postnatal corticosteroid therapy not be used routinely in preterm infants at risk for BPD (extremely preterm (EPT) infants $<28$ weeks gestation as known adverse effects overweigh potential benefits especially association with increased risk of cerebral palsy (CP) and abnormal neurologic examination at long-term follow-up [7]. Similar findings were reported in a recent systemic review wherein the authors concluded that despite the fact that some studies reported a modulating effect of treatment regimens in favor of higher dosage regimens on the incidence of BPD and neuro-developmental impairment, recommendations on the optimal type of corticosteroid, the optimal dosage, or the optimal timing of initiation for the prevention of BPD in preterm infants cannot be made based on the current level of evidence [8]. 
- Other Routes of administration: inhaled corticosteroids have not been found to be beneficial in prevention of BPD and hence not recommended [9]. A systematic review and meta-analysis including two clinical trials concluded that intra-tracheal administration of budesonide-surfactant combination was associated with decreased incidence of BPD alone or composite outcome of death or BPD in VLBW infants [10]. Another meta-analysis with trial sequential analysis included twenty five RCTs $(n=3249)$ and estimated a lower risk of the primary outcome of BPD and composite outcome of death or BPD in airway administration of corticosteroids (AACs) group (inhalation or instillation) than placebo. The authors reported that AACs (especially instillation of budesonide using surfactant as a vehicle) is equivalent to systemic corticosteroids for preventing BPD [11]. However, unless the results are corroborated in larger trials, this route is not recommended for use for prevention of BPD.

- Restrictive use of corticosteroids: Restrictive use of systemic corticosteroids in extremely premature infants (EPT) who remain ventilator/oxygen dependent at postnatal age 3 to 4 weeks or late preterm infants with established BPD who are ventilator/oxygen dependent is successfully used at some centers based on limited data.

\section{Caffeine}

- Mechanism of action: Caffeine is a methyxanthine which mainly acts as a competitive adenosine receptor antagonist resulting in stimulation of medullary respiratory center, increased sensitivity to carbon dioxide and stimulation of diaphragmatic contractility [12].

- Basis of use in BPD: In addition to shortening of ventilation time by stimulation of respiratory center, caffeine is known to exert diuretic effects [13], provides neuronal injury protection [14] and induces surfactant protein B (SP-B) transcription [15]. Recently, growing evidence has suggested that caffeine can inhibit the secretion of proinflammatory cytokines, especially IL-1 $\beta$, to reduce inflammation in hyperoxia and lipopolysaccharide (LPS)-induced rodent models [16]. Dumpa V., et al. have proposed that postnatal caffeine modulates angiogenic gene expression early in lung development in male mice following hyperoxia induced lung injury and that it may restore the pulmonary microvasculature and alveolarization in adult lung [17]. Recently, nucleotide-binding domain and leucine-rich repeat protein 3 (NLRP3) inflammasome activation has been demonstrated to be essential for the pathogenesis of BPD and in a study by Zhao W., et al. it was concluded that caffeine inhibits NLRP3 inflammasome activation in lipopolysaccharide (LPS) induced human monocyte leukemia cell line (THP-1) macrophages [18]. Lung function is improved in infants born under 31 weeks of gestation when treated with caffeine. Results from a recent study by Sanchez-Solis M., et al. show that there is an early beneficial effect of caffeine treatment on lung function (FVC and $\mathrm{FEV}_{0.5}$ ) in infant born under 31 weeks of gestation who did not suffer from BPD [19].

- Route of administration: Intravenous or orally.

- $\quad$ Side effects of Caffeine: No significant short or long-term adverse effects of caffeine therapy in the neonates have been reported in the CAP trial and its subsequent reports of outcomes when used for apnoea of prematurity [20]. In RCTs using high-dose caffeine therapy, the initial slow rate of growth and clinically insignificant tachycardia were the only reported side effects.

- Current Status: Caffeine is recommended for use in prevention of BPD among preterm neonates. In a recent systematic review and meta-analysis, it was reported that earlier initiation of caffeine, compared to later, was associated with a decreased risk of BPD (5 observational studies; $n=63,049$, adjusted OR 0.69; 95\% CI 0.64-0.75, GRADE: low quality). High-dose caffeine, compared to standard-dose, was associated with a decreased risk of BPD ( 3 randomized trials, $n=432$, OR 0.65; 95\% CI 0.43-0.97; GRADE: low quality) [21]. Similar results were reported in another systematic review and meta-analysis by Park HW., et al. [22].

Non-invasive ventilation strategies

Current Status: AA systematic review and meta-analysis of Association of Noninvasive Ventilation Strategies With Mortality and Bronchopulmonary Dysplasia Among Preterm Infants compared seven ventilation strategies including nasal continuous positive airway pressure (CPAP) alone, intubation and surfactant administration followed by immediate extubation (INSURE), less invasive surfactant administration (LISA), noninvasive intermittent positive pressure ventilation, nebulized surfactant administration, surfactant administration via laryngeal mask airway, and mechanical ventilation concluded that the use of LISA was associated with the lowest likelihood of the composite outcome of death or BPD at 36 weeks' postmenstrual age (low quality evidence) [23]. Restricted ventilation has also been associated with reduced neurodevelopmental impairment in preterm infants assessed at 24 months corrected age [24]. Avoidance of endotracheal mechanical ventilation (eMV) (nine RCTs, 3486 infants) reduced the risk of death or BPD, with a risk ratio of 0.90 (95\% CI 0.84-0.97) and a number needed to treat of 35 in a meta-analysis done by Fischer HS., et al. [25] Sustained inflations of pressure during mechanical ventilation in very preterm infants had no effect and may even increase mortality in very preterm infants in comparison to intermittent pressure ventilation strategies. A recent multicentric RCT was halted for increased early mortality in the sustained inflations arm [26]. A review based on recent metanalysis reported that interventions with proven effectiveness in reducing BPD include the primary use of non-invasive respiratory support, the application of surfactant without endotracheal ventilation and the use of volume-targeted ventilation in infants requiring 
endotracheal intubation. Following extubation, synchronised nasal ventilation is more effective than continuous positive airway pressure in reducing BPD [27]

\section{Vitamin A}

- Basis of use in BPD: Retinoic acid plays a key role in lung development improving alveolar septation and preterms have been found to be deficient in Vitamin A. Furthermore, retinoids have been documented to control surfactant phospholipid biosynthesis.

- Route of administration: Intramuscular

- Current Status: VA has been identified as a strategy to decrease the incidence of BPD. A systematic review and meta-analysis by Araki S on the role of Vitamin A (VA) in prevention of bronchopulmonary dysplasia in extremely low birth weight infants including four studies (total, 1,011 infants) with VA administered intramuscularly in 3 studies and orally in 1 study depicted that VA supplementation for ELBWIs had benefited oxygen dependency at the postmenstrual age of 36 weeks in survivors (pooled risk ratio, $0.88 ; 95 \%$ confidence intervals (CI), 0.77-0.99; 4 trials, 841 infants, moderate certainty of evidence), which is similar to the meta-analysis in VLBWIs. Length of hospital stay was reduced in the VA group (mean difference, -49.9; $95 \%$ CI, -88.78 to $-11.02 ; 1$ trial, 20 infants, low certainty of evidence). The meta-analysis showed no reduction in the risk of neonatal death, oxygen use at 28 days in survivors, duration of mechanical ventilation, intraventricular hemorrhage, retinopathy of prematurity and necrotizing enterocolitis [28]. Similar observations were shared in another systematic review which included two RCTs. The review concluded that there was statistically significant reduction in the incidence of BPD (oxygen requirement at 36 weeks of postmenstrual age (PMA)) (relative risk (RR) 0.88; 95\%CI 0.77-0.99; p=.04; NNTB 14) and borderline significant reduction in combined outcomes of mortality/ BPD (oxygen requirement at 36 weeks of PMA) (RR 0.90; 95\%CI 0.82-1.00; $\mathrm{p}=.05)$. However, oxygen requirement at 28 days of life and combined outcome of mortality/BPD (oxygen requirement at 28 days of life) were not statistically significant [29]. Initial large-scale prospective evaluations have shown clear benefit of VA in reducing the incidence of CLD or death. However, changing definitions of BPD and implementation of noninvasive ventilation strategies limit the application of early studies. During a drug shortage, VA supply and use declined dramatically, but BPD rates remained stable. With concerns of sepsis and necrotizing enterocolitis in small-scale studies, and in light of the limited evidence, further evaluations are necessary before VA can be recommended as a cornerstone of BPD prevention.
Macrolide antibiotics

- Mechanism of action: Macrolides exert their antibiotic effect by binding irreversibly to the 50 s subunit of bacterial ribosomes thereby inhibiting translocation of tRNA during translation. This action is mainly bacteriostatic but macrolides can be bactericidal in high concentrations.

- Basis of use in BPD: Respiratory tract colonization with the genital mycoplasma species Urea plasma parvum and Ureaplasma urealyticum in preterm infants is reported to be a significant risk factor for bronchopulmonary dysplasia (BPD) [30]. Azithromycin (AZ) and clarithromycin are preferentially concentrated in lung epithelial lining fluid and alveolar macrophages. These have immunomodulatory function and antimicrobial activity against urea plasma as documented in vivo experimental models [31]. Treatment with these antibiotics may enhance urea plasma clearance in infected infants and inhibit the pulmonary inflammatory response possibly contributing to a decreased risk for BPD [32]. A new antimicrobial, Solithromycin has shown promising efficacy in sheep models [33].

- Route of administration: Intravenous.

- Adverse effects: Arrhythmogenic and hence caution advised.

- Current status: In patients with preterm premature rupture of membrane (pPROM) between 22 and 27weeks of gestation, the administration of azithromycin may improve the perinatal outcomes [34]. A meta-analysis including six studies involving 469 preterm infants demonstrated that macrolides when used prophylactically (4 studies) did not show significant reduction in BPD (risk ratio, RR, 0.88, 95\% confidence interval, CI, 0.75-1.03), death (RR 0.89, 95\% CI 0.79-1.01) or in the composite outcome of BPD/death. Similarly, there was no significant reduction in BPD (RR $0.64,95 \%$ CI $0.31-1.31$ ) or the composite outcome of BPD/ death (RR 0.41, 95\% CI 0.05-3.13), when macrolides were used in ureaplasma-positive infants. However, prophylactic azithromycin therapy (3 studies) was associated with significant reduction in BPD (RR 0.83, 95\% CI 0.71-0.97; number needed to treat, NNT, 10) and composite outcome of BPD/death (RR 0.86, 95\% CI 0.77-0.97; NNT 10). The review concluded that prophylactic azithromycin therapy was associated with statistically significant reduction in BPD and the composite outcome of BPD/death in preterm infants [35]. A recent retrospective case-control study of preterm babies delivered at $\leq 30$ weeks of gestational age (GA) from 2012 to 2016 reported that maternal ureaplasma colonization was associated with moderate-severe BPD despite the use of azithromycin treatment. In addition, if the neonatal respiratory tract was colonized, then moderatesevere BPD developed even with maternal AZ treatment. Hence, selective antenatal and postnatal treatment of 
ureaplasma colonization would be needed to control BPD development [36]. Another systematic review documented that azithromycin significantly reduced the risk of BPD in extremely premature neonates $(\mathrm{RR}=0.83,95 \%$ CI 0.71 to $0.98, \mathrm{p}=0.02)$. There was no significant difference in the incidence of elevated liver enzymes between the azithromycin and placebo group $(p=0.76)$. There were four cases of infantile hypertrophic pyloric stenosis (IHPS) [37]. However, before routine use of azithromycin is recommended in the neonatal population further studies are needed especially related to safety profile and long term neurodevelopmental outcomes.

\section{Nitric oxide}

- Mechanism of action: Short-lived free radical that has role in macrophage activation in a wound, cytokine synthesis and proliferation of fibroblasts, epithelization, collagen synthesis and wound healing.

- $\quad$ Basis of use in BPD: Inhaled Nitric oxide (iNO) has been reported to prevent right ventricle hypertrophy and pulmonary vascular remodeling in Bleomycin induced BPD like changes in neonatal rat lungs resulting in partial improvement of lung structure [38]. It has been reported to decrease pulmonary vascular resistance. NO-mediated biosynthetic pathways considered to be contributor to the pathogenesis of BPD were shown to be corrected by use of iNO [39].

- Route of administration: Inhalational.

- Contraindication: Neonates dependent with ductaldependent congenital heart disease.

- Adverse effects: Hypotension, withdrawal manifestations (e.g., increased pulmonary artery pressure, decreased $\mathrm{PaO}_{2}$, increase in or return to right-to-left shunting of blood, atelectasis), hematuria, and methemoglobinemia.

- Current status: Multiple clinical trials have examined the use of iNO in preterm infants to prevent BPD and have concluded that iNO does not have unequivocal benefit in this at-risk population [40]. It is suggested that inhaled ethyl nitrite could be a superior therapeutic agent than iNO because it more efficiently S-nitrosylates proteins, does not create potentially harmful by-products like peroxynitrite, and improves tissue blood flow and oxygen delivery. In 2010, the NIH Consensus Development Conference provided therapeutic guidelines for the use of iNO; their primary conclusion is that apart from occasional instances of pulmonary hypertension or hypoplasia, routine or rescue use of iNO cannot be recommended at this time in preterm infants [41].

\section{Fluid restriction and diuretic therapy}

Fluid restriction is often recommended as part of the management of infants with early or established bronchopulmonary dysplasia (BPD). However, there is no evidence to support the practice of fluid restriction in infants with early or established
BPD [42]. A case series studied the usefulness of angiotensinconverting enzyme (ACE) inhibition by measuring clinical and echocardiographic improvements in six consecutive infants with severe BPD unresponsive to conventional therapy. Improvements were observed for both systolic and diastolic cardiac function which was accompanied by increased pulmonary vein flow. Right ventricular output increased accompanied by a significant lowering of pulmonary vascular resistance [43]. However, further larger studies are needed to study the role of ACE inhibition in BPD.

\section{Emerging strategies}

The growing understanding of BPD pathogenesis is prompting researchers to evaluate newer preventive strategies. The use of mesenchymal stem cells (MSC) is one such strategy. A first human clinical trial of MSC treatment for BPD was performed as a phase I dose-escalation trial using umbilical cord blood-derived MSC which demonstrated short and long-term safety and feasibility of MSC based on significantly reduced inflammatory marker expression observed in tracheal aspirates [44]. Currently several clinical trials of MSC use for BPD are ongoing to investigate the efficacy of MSC in the prevention or treatment of BPD in premature infants [45]. Even if clinical trials of MSC for BPD are successful, substantial challenges will still exist in translating this therapy into clinical use. One of the main factors that complicate the selection of MSC for use is the heterogeneity of MSC populations [46]. Other issues related to largescale production, logistics, and transport of MSC and development of cell-free preparations remains a major problem [47]. Recent studies demonstrated that connective tissue growth factor (CTGF) is involved in the development of BPD in experimental models Conditional overexpression of CTGF in alveolar epithelial type II cells disrupts alveolarization and vascular development, induces vascular remodeling, and results in pulmonary hypertension, the pathological hallmarks of severe BPD which can be prevented by inhibition of CTGF by a monoclonal antibody as demonstrated in a rodent model of BPD induced by hyperoxia. CTGF may thus be a novel target for BPD therapy in preterm infants [48]. Role of various other preventive strategies is being studied by various researchers like use of nebulized peroxysome proliferator activated receptor gamma (PPAR $\gamma$ ) agonists and sildenafil $[49,50]$

\section{Conclusion}

Bronchopulmonary dysplasia (BPD) is leading cause of morbidity and mortality among preterm neonates. With changing obstetric care and neonatal management protocols, profile of BPD is changing and has led to numerous modifications in BPD definition. Interventions with proven effectiveness in reducing BPD include the primary use of non-invasive respiratory support, early commencement of caffeine citrate and restrictive use of intravenous corticosteroids. Intramuscular Vitamin A, intratracheal administration of budesonide with surfactant and azithromycin especially in ureaplasma colonized neonates look promising but need further exploration before being routinely recommended for prevention of BPD in premature neonates. Newer therapies like 
mesenchymal stem cells, inhibition of connective tissue growth factor (CTGF) by a monoclonal antibody, nebulized peroxysome proliferator activated receptor gamma (PPAR $\gamma$ ) agonists and sildenafil are currently being studied for their potential role in BPD prevention.

\section{Bibliography}

1. Northway WH., et al. "Pulmonary disease following respirator therapy of hyaline-membrane disease, bronchopulmonary dysplasia". The New England Journal of Medicine 276 (1967): 357-368.

2. Jobe AH and Bancalari E. "Bronchopulmonary dysplasia". American Journal of Respiratory and Critical Care Medicine 163.17 (2001): 1723-1729.

3. Higgins RD., et al. "Bronchopulmonary Dysplasia: Executive Summary of a Workshop". The Journal of Pediatrics 197 (2018): 300-308.

4. Bancalari E and Jain D. "Bronchopulmonary Dysplasia: Can We Agree on a Definition?" American Journal of Perinatology 35.6 (2018): 537-540.

5. Greaves MW. "Anti-inflammatory action of corticosteroids". Postgraduate Medical Journal 52.612 (1976): 631-633.

6. Aulakh R and Singh S. "Strategies for minimizing corticosteroid toxicity: A Review”. The Indian Journal of Pediatrics 75 (2008): 1067.

7. Watterberg KL. "American Academy of Pediatrics. Committee on Fetus and Newborn. Policy statement--postnatal corticosteroids to prevent or treat bronchopulmonary dysplasia". Pediatrics 126.4 (2010): 800-808.

8. Wes Onland., et al. "Systemic corticosteroid regimens for prevention of bronchopulmonary dysplasia in preterm infants". Cochrane Database of Systematic Reviews 1 (2017): CD010941.

9. Shah SS., et al. "Inhaled versus systemic corticosteroids for the treatment of bronchopulmonary dysplasia in ventilated very low birth weight preterm infants". Cochrane Database of Systematic Reviews 10 (2017): CD002057.

10. Venkataraman R., et al. "Intratracheal Administration of Budesonide-Surfactant in Prevention of Bronchopulmonary Dysplasia in Very Low Birth Weight Infants: A Systematic Review and Meta-Analysis". Pediatric Pulmonology 52.7 (2017): 968-975.

11. Zhang ZQ., et al. "Airway administration of corticosteroids for prevention of bronchopulmonary dysplasia in premature infants: a meta-analysis with trial sequential analysis". BMC Pulmonary Medicine 17.1 (2017): 207.

12. Jain D and Bancalari E. "Prevention of bronchopulmonary dysplasia: current strategies". Zhongguo Dang Dai Er Ke Za Zhi 19.8 (2017): 841-851.
13. Pacifici GM. "Clinical pharmacology of theophylline in preterm infants: effects, metabolism and pharmacokinetics". Current Pediatric Reviews 10.4 (2014): 297-303.

14. Endesfelder S., et al. "Neuroprotection by Caffeine in Hyperoxia-Induced Neonatal Brain Injury". International Journal of Molecular Sciences (2017): 18.

15. Fehrholz M., et al. "Amplification of steroid-mediated SP-B expression by physiological levels of caffeine". American Journal of Physiology-Lung Cellular and Molecular Physiology 306.1 (2014): L101-109.

16. Lodha A., et al. "Association of early caffeine administration and neonatal outcomes in very preterm neonates". JAMA Pediatrics 169.1 (2015): 33-38.

17. Dumpa V., et al. "Caffeine is associated with improved alveolarization and angiogenesis in male mice following hyperoxia induced lung injury". BMC Pulmonary Medicine 19.1 (2019):138.

18. Zhao W., et al. "Caffeine Inhibits NLRP3 Inflammasome Activation by Suppressing MAPK/NF-KB and A2aR Signaling in LPS-Induced THP-1 Macrophages". International Journal of Biological Sciences 15.8 (2019):1571-1581.

19. Sanchez-Solis M., et al. "Impact of early caffeine therapy in preterm newborns on infant lung function". Pediatric Pulmonology (2019).

20. Schmidt B., et al. "Caffeine therapy for apnea of prematurity". The New England Journal of Medicine 354 (2006): 2112-2121.

21. Pakvasa MA., et al. "Optimizing Caffeine Use and Risk of Bronchopulmonary Dysplasia in Preterm Infants: A Systematic Review, Meta-analysis, and Application of Grading of Recommendations Assessment, Development, and Evaluation Methodology". Clinics in Perinatology 45.2 (2018): 273-291.

22. Park HW., et al. "Early Caffeine Use in Very Low Birth Weight Infants and Neonatal Outcomes: A Systematic Review and Meta-Analysis". Journal of Korean Medical Science 30.12 (2015):1828-1835.

23. Isayama T., et al. "Association of Noninvasive Ventilation Strategies with Mortality and Bronchopulmonary Dysplasia Among Preterm Infants: A Systematic Review and Metaanalysis". The Journal of the American Medical Association 316.6 (2016): 611-624.

24. Vliegenthart RJS., et al. "Restricted Ventilation Associated with Reduced Neurodevelopmental Impairment in Preterm Infants". Neonatology 112.2 (2017): 172-179.

25. Fischer HS., et al. "Sustained inflations and avoiding mechanical ventilation to prevent death or bronchopulmonary dysplasia: a meta-analysis". The European Respiratory Review 27.150 (2018):180083. 
26. Kirpalani H., et al. "The International "Sustained aeration for infant lung" (SAIL) randomized trial”. In Pediatric Academic Societies Meeting (2018): 5-8.

27. Poets CF and Lorenz L. "Prevention of bronchopulmonary dysplasia in extremely low gestational age neonates: current evidence". Archives of Disease in Childhood Fetal and Neonatal Edition 103.3 (2018): F285-F291.

28. Araki S., et al. "Vitamin A to prevent bronchopulmonary dysplasia in extremely low birth weight infants: a systematic review and meta-analysis". PLoS one 13.11 (2018): e0207730.

29. Garg BD., et al. "Role of vitamin A supplementation in prevention of bronchopulmonary dysplasia in extremely low birth weight neonates: a systematic review of randomized trials". The Journal of Maternal-Fetal and Neonatal Medicine 32.15 (2019): 2608-2615.

30. Lowe J., et al. "Association between pulmonary ureaplasma colonization and bronchopulmonary dysplasia in preterm infants: updated systematic review and meta-analysis". The Pediatric Infectious Disease Journal 33.7 (2014): 697-702.

31. Acosta EP., et al. "Transplacental transfer of Azithromycin and its use for eradicating intra-amniotic ureaplasma infection in a primate model". The Journal of Infectious Diseases 209 (2014): 898-904.

32. Waites KB., et al. "Comparative in vitro susceptibilities of human mycoplasmas and ureaplasmas to a new investigational ketolide, CEM-101". Antimicrobial Agents and Chemotherapy 53 (2009): 2139-2141.

33. Keelan JA., et al. "Maternal administration of solithromycin, a new, potent, broad-spectrum fluoroketolide antibiotic, achieves fetal and intra-amniotic antimicrobial protection in a pregnant sheep model". Antimicrobial Agents and Chemotherapy 58 (2014): 447-454.

34. Tanaka S., et al. "New antibiotic regimen for preterm premature rupture of membrane reduces the incidence of bronchopulmonary dysplasia". Journal of Obstetrics and Gynaecology Research 45.5 (2019): 967-973.

35. Nair V., et al. "Azithromycin and other macrolides for prevention of bronchopulmonary dysplasia: a systematic review and meta-analysis". Neonatology 106.4 (2014): 337347.

36. Kim SH., et al. "Effect of antenatal azithromycin for Ureaplasma spp. on neonatal outcome at $\leq 30$ weeks' gestational age". Pediatrics International 61.1 (2019): 58-62.

37. Smith C., et al. "Use and safety of azithromycin in neonates: a systematic review". BMJ Open 5.12 (2015): e008194.

38. Tourneux P., et al. "Inhaled nitric oxide improves lung structure and pulmonary hypertension in a model of bleomycin-induced bronchopulmonary dysplasia in neonatal rats". The American Journal of Physiology-Lung Cellular and Molecular Physiology 297.6 (2009): L1103-1111.
39. McCurnin DC., et al. "Inhaled NO improves early pulmonary function and modifies lung growth and elastin deposition in a baboon model of neonatal chronic lung disease". The American Journal of Physiology-Lung Cellular and Molecular Physiology 288.3 (2005): L450-459.

40. Kilbride H., et al. "Childhood Pulmonary Function, Exercise Capacity, and Exhaled Nitric Oxide Levels: Outcomes following Neonatal Treatment with Inhaled Nitric Oxide to Prevent Bronchopulmonary Dysplasia". American Journal of Perinatology 36.4 (2019): 360-365.

41. Raffay TM., et al. "Can nitric oxide-based therapy prevent bronchopulmonary dysplasia?" Clinics in Perinatology 39.3 (2012): 613-638.

42. Barrington KJ., et al. "Fluid restriction for treatment of preterm infants with chronic lung disease". Cochrane Database of Systematic Reviews 2 (2017): CD005389.

43. Sehgal A., et al. "ACE inhibition for severe bronchopulmonary dysplasia - an approach based on physiology". Physiological Reports 6.17 (2018): e13821.

44. ChangYS., etal. "Mesenchymal stem cells for bronchopulmonary dysplasia: Phase 1 dose-escalation clinical trial". The Journal of Pediatrics 164 (2014): 966-972.e966.

45. Namba F. "Mesenchymal stem cells for the prevention of bronchopulmonary dysplasia". Pediatrics International 61.10 (2019): 945-950.

46. Morita Y., et al. "Heterogeneity and hierarchy within the most primitive hematopoietic stem cell compartment". The Journal of Experimental Medicine 207 (2010): 1173-1182.

47. Fung ME and Thebaud B. "Stem cell-based therapy for neonatal lung disease: It is in the juice". Pediatric Research 75 (2014): 2-7.

48. Wang X., et al. "CTGF: A potential therapeutic target for Bronchopulmonary dysplasia". European Journal of Pharmacology 860 (2019):172588.

49. Lecarpentier Y., et al. "Bronchopulmonary Dysplasia: Crosstalk Between PPAR $\gamma$, WNT/ $\beta$-Catenin and TGF- $\beta$ Pathways the Potential Therapeutic Role of PPAR $\gamma$ Agonists". Frontiers in Pediatrics 3.7 (2019): 176.

50. Abounahia FF., et al. "Prophylactic Sildenafil in Preterm Infants at Risk of Bronchopulmonary Dysplasia: A Pilot Randomized, Double-Blinded, Placebo-Controlled Trial". Clinical Drug Investigation 39.11 (2019): 1093-1107.

Volume 2 Issue 12 December 2019 C All rights are reserved by Roosy Aulakh. 